\title{
Spirometric values for normal adult males in Greece
}

\author{
N. D. POURNARAS, X. KONDAKIS, and \\ D. PAPA NAST ASSIOU
}

Laboratory of Ergospirometry, King Paul Hospital, Athens (609), Greece

\begin{abstract}
We have measured the vital capacity and forced expiratory volume in 1 second of 50 healthy men aged 20 to 25 and 50 aged 55 to 60 years in order to obtain normal values for reference use for pulmonary tests in Greece. We constructed tables of mean and $95 \%$ confidence limits for ages 20 to 70 and height 1.5 to 1.9 metres, assuming that these respiratory values vary linearly with age and height. The differences in our results from those obtained in the United States of America and in France show that misinterpretation of pulmonary function tests may result from inappropriate reference values.
\end{abstract}

For several years the normal values for vital capacity (VC) and forced expiratory volume in one second $\left(\mathrm{FEV}_{1}\right)$ used as reference standards in Greece were those of Baldwin, Cournand, and Richards (1948). Data obtained more recently in men of European descent from the U.S.A. (Kory, Callahan, Boren, and Syner, 1961), Britain (Cotes, Higgins, Rossiter, and Wilton, 1964; Cotes, 1965), and Sweden (Berglund et al., 1963) are similar. Those obtained from France, however (Commission des Communautés Européennes, 1967), show considerable differences. We therefore considered it necessary to establish normal reference values for Greece, where different genetic and environmental conditions prevail from those in Western Europe and the U.S.A., and the present work was undertaken for that purpose.

\section{MATERIAL AND METHODS}

We studied a group of 50 men aged 20 to 25 years and another group of 50 aged 55 to 60 years. Most of the subjects in the younger age group were medical students. The older group included various professions, the white-collar employee being predominant. All subjects were selected on two criteria : (a) absence of pulmonary symptoms (cough, sputum, and dyspnoea) and (b) absence, within the previous three years, of a chest illness causing time off work. None of the subjects gave a history of cough and sputum on most days for as much as three months in the year. The smoking habits of the subjects were not taken into consideration because in previous studies (Cotes et al., 1964) it was established that subdivision of the subjects according to their tobacco consumption revealed no significant differences in FEV.
We measured the VC and the $\mathrm{FEV}_{1}$ with a Mac- $\vec{\theta}$ Dermott dry spirometer designed and manufactured N at the Pneumoconiosis Research Unit of the Medical Research Council. The apparatus is self-correcting for B.T.P.S. The overall calibration of the apparatus was tested frequently by the method described by McKerrow, McDermott, and Gilson (1960) and was found to be unaltered throughout the studies. $\Omega$ Each subject was asked to perform four consecutive $\overrightarrow{\vec{F}}$ tests and the result of the highest was recorded. In 윽 almost all the subjects no differences were noted between the efforts. The standing height in bare feet was measured.

Regressions were calculated between standing height and respiratory values in each age group separately. The mean values of $\mathrm{VC}$ and $\mathrm{FEV}_{1}$ as $\stackrel{0}{x}$ well as the corresponding lower level of the $95 \%$ confidence limits-as applied to individual valueswere calculated for each height, in steps of $5 \mathrm{~cm}$, $\delta$ from $1.5 \mathrm{~m}$ to $1.9 \mathrm{~m}$ as the decrease in respiratory values with age is known to be closely linear (Cotes, 0 1965). The expected values for other ages, intermediate to the above age groups, were calculated by $\frac{D}{O}$ linear interpolation, which was applied on both the mean and the lower confidence interval limit. The $N$ body-weight of the subjects was not taken into consideration (Kory et al., 1961).

\section{RESULTS}

The mean heights of the two age groups (Table I) differ significantly $(\mathrm{P}<0.01)$ but their variances? are almost identical. The variances of $\mathrm{VC}$ and ${ }^{\circ}$ $\mathrm{FEV}_{1}$ do not differ significantly between the two $\frac{\vec{P}}{\mathbb{D}}$ groups. There is a statistically significant difference $\stackrel{\odot}{\oplus}$

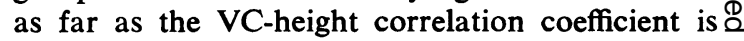
concerned $(P<0.05)$, in contrast to the $F E V_{1}-\frac{O}{2}$ 
T A B LE I

SPIROMETRIC VALUES AND STATISTICAL FEATURES OF TWO AGE GROUPS

\begin{tabular}{|c|c|c|}
\hline & \multicolumn{2}{|c|}{ Age Group } \\
\hline & $\begin{array}{l}20-25 \\
n=50\end{array}$ & $\begin{array}{l}55-60 \\
n=50\end{array}$ \\
\hline $\begin{array}{l}\text { Height (metres) } \\
\text { Vital capacity (litres) } \\
\text { Coefficient of variation } \\
\text { FEV }_{1} \text { (litres) } \\
\text { Coefficient of variation } \\
\text { Correlation coefficient }\end{array}$ & $\begin{array}{c}1.74 \quad 0.062 \\
5.430 .588 \\
11 \% \% \\
4.77 \quad 0.511 \\
11 \%\end{array}$ & $\begin{array}{c}1.64 \quad 0.005 \\
3.920 .595 \\
15 \% \\
2.950 .482 \\
16 \%\end{array}$ \\
\hline $\begin{array}{l}\text { VC-Height } \\
\text { FEV } \\
\text { Regressiont }\end{array}$ & $\begin{array}{l}0.77 \\
0.73\end{array}$ & $\begin{array}{l}0.52 \\
0.61\end{array}$ \\
\hline $\begin{array}{l}\text { VC-Height } \\
\text { Seb } \\
\text { FEV }_{1} \text {-Height } \\
\text { Seb }\end{array}$ & $\begin{array}{c}7 \cdot 326 \mathrm{H}_{\mathrm{m}}-7 \cdot 34 \\
0 \cdot 375 \\
6 \cdot 025 \mathrm{H}_{\mathrm{m}}-5 \cdot 73 \\
0 \cdot 349\end{array}$ & $\begin{array}{c}5 \cdot 601 \mathrm{H}_{m}-5 \cdot 27 \\
0 \cdot 411 \\
5 \cdot 364 \mathrm{H}_{m-5} \cdot 87 \\
0.386\end{array}$ \\
\hline
\end{tabular}

height correlation coefficient in which no such difference exists. The regression coefficients do not differ between the two groups and the regression equations are similar. In the 20 to 25 year age group the regression of $\mathrm{VC}$ on height was $7 \cdot 326 \mathrm{H}_{\mathrm{m}}-7 \cdot 34$, that of the 55 to 60 year age group being $5 \cdot 601 \mathrm{H}_{m}-5 \cdot 27$. The regression of $\mathrm{FEV}_{1}$ on height was respectively $6.025 \mathrm{H}_{m}-5.73$ and $5 \cdot 364 \mathrm{H}_{\mathrm{m}}-5 \cdot 87$.

Table II presents the expected values which can be considered as normal in Greece as well as their lower $95 \%$ confidence limits. Thus an individual with spirometric values lower than these limits has a $2.5 \%$ probability of being normal.

T A B L E I I

NORMAL REFERENCE VALUES (LITRES) VC AND FEV ${ }_{1}$ IN GREECE IN RELATION TO AGE AND HEIGHT (a) Vital Capacity

\begin{tabular}{|c|c|c|c|c|c|c|c|c|c|c|}
\hline \multirow{2}{*}{$\begin{array}{l}\text { Height } \\
\text { (m) }\end{array}$} & \multicolumn{10}{|c|}{ Age Group } \\
\hline & $20-25$ & $26-30$ & $31-35$ & $36-40$ & $41-45$ & $46-50$ & $51-55$ & $56-60$ & $61-65$ & $66-70$ \\
\hline $\begin{array}{l}1.50 \\
155 \\
1.60 \\
1.65 \\
1.70 \\
1.75 \\
1.80 \\
1.85 \\
1.90\end{array}$ & $\begin{array}{c}3 \cdot 65 \\
2 \cdot 78 \\
4 \cdot 02 \\
3 \cdot 19 \\
4 \cdot 38 \\
3 \cdot 58 \\
4 \cdot 74 \\
3 \cdot 98 \\
5 \cdot 11 \\
4 \cdot 35 \\
5 \cdot 48 \\
4 \cdot 72 \\
5 \cdot 84 \\
5 \cdot 08 \\
6 \cdot 21 \\
5 \cdot 42 \\
6 \cdot 58 \\
5 \cdot 77\end{array}$ & $\begin{array}{c}3 \cdot 58 \\
2 \cdot 71 \\
3 \cdot 94 \\
3 \cdot 11 \\
4 \cdot 28 \\
3 \cdot 47 \\
4 \cdot 63 \\
3 \cdot 86 \\
4 \cdot 99 \\
4 \cdot 22 \\
5 \cdot 35 \\
4 \cdot 57 \\
5 \cdot 69 \\
4 \cdot 91 \\
6 \cdot 05 \\
5 \cdot 23 \\
6 \cdot 40 \\
5 \cdot 57\end{array}$ & $\begin{array}{c}3 \cdot 50 \\
2 \cdot 63 \\
3 \cdot 58 \\
3 \cdot 02 \\
4 \cdot 18 \\
3 \cdot 37 \\
4 \cdot 52 \\
3 \cdot 74 \\
4 \cdot 86 \\
4 \cdot 08 \\
5 \cdot 21 \\
4 \cdot 42 \\
5 \cdot 54 \\
4 \cdot 74 \\
5 \cdot 89 \\
5 \cdot 05 \\
6 \cdot 23 \\
5 \cdot 37\end{array}$ & $\begin{array}{c}3 \cdot 43 \\
2 \cdot 56 \\
3 \cdot 77 \\
2 \cdot 94 \\
4 \cdot 08 \\
3 \cdot 27 \\
4 \cdot 41 \\
3 \cdot 62 \\
4 \cdot 74 \\
3 \cdot 95 \\
5 \cdot 08 \\
4 \cdot 27 \\
5 \cdot 39 \\
4 \cdot 57 \\
5 \cdot 73 \\
4 \cdot 87 \\
6 \cdot 08 \\
5 \cdot 17\end{array}$ & $\begin{array}{c}3 \cdot 35 \\
2 \cdot 48 \\
3 \cdot 68 \\
2 \cdot 85 \\
3 \cdot 98 \\
3 \cdot 17 \\
4 \cdot 30 \\
3 \cdot 50 \\
4 \cdot 61 \\
3 \cdot 82 \\
4 \cdot 94 \\
4 \cdot 12 \\
5 \cdot 25 \\
4 \cdot 40 \\
5 \cdot 57 \\
4 \cdot 68 \\
5 \cdot 90 \\
4.97\end{array}$ & $\begin{array}{c}3 \cdot 28 \\
2 \cdot 41 \\
3 \cdot 59 \\
2 \cdot 76 \\
3 \cdot 88 \\
3 \cdot 06 \\
4 \cdot 19 \\
3 \cdot 38 \\
4 \cdot 49 \\
3 \cdot 68 \\
4 \cdot 80 \\
3 \cdot 97 \\
5 \cdot 10 \\
4 \cdot 23 \\
5 \cdot 41 \\
4 \cdot 50 \\
5 \cdot 72 \\
4 \cdot 77\end{array}$ & $\begin{array}{c}3 \cdot 20 \\
2 \cdot 32 \\
3 \cdot 50 \\
2 \cdot 67 \\
3 \cdot 78 \\
2 \cdot 96 \\
4 \cdot 08 \\
3 \cdot 26 \\
4 \cdot 38 \\
3 \cdot 55 \\
4 \cdot 67 \\
3 \cdot 82 \\
4 \cdot 96 \\
4 \cdot 06 \\
5 \cdot 25 \\
4 \cdot 32 \\
5 \cdot 54 \\
4 \cdot 57\end{array}$ & $\begin{array}{c}3 \cdot 13 \\
2 \cdot 24 \\
3 \cdot 41 \\
2 \cdot 58 \\
3 \cdot 69 \\
2 \cdot 86 \\
3 \cdot 97 \\
3 \cdot 14 \\
4 \cdot 25 \\
3 \cdot 42 \\
4 \cdot 53 \\
3 \cdot 66 \\
4 \cdot 81 \\
3 \cdot 90 \\
5 \cdot 09 \\
4 \cdot 13 \\
5 \cdot 37 \\
4 \cdot 36\end{array}$ & 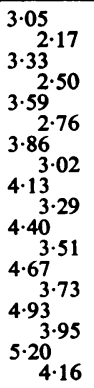 & $\begin{array}{c}2 \cdot 98 \\
2.09 \\
3 \cdot 24 \\
2.41 \\
3 \cdot 50 \\
2.66 \\
3 \cdot 75 \\
2.90 \\
4 \cdot 00 \\
3 \cdot 15 \\
4 \cdot 26 \\
3 \cdot 36 \\
4 \cdot 53 \\
3.56 \\
4 \cdot 77 \\
3 \cdot 76 \\
5 \cdot 02 \\
3.96\end{array}$ \\
\hline
\end{tabular}

(b) Forced Expiratory Volume (FEV $)$

\begin{tabular}{|c|c|c|c|c|c|c|c|c|c|c|}
\hline \multirow{2}{*}{$\underset{\text { (m) }}{\text { Height }}$} & \multicolumn{10}{|c|}{ Age Group } \\
\hline & $20-25$ & $26-30$ & $31-35$ & $36-40$ & $41-45$ & $46-50$ & $51-55$ & $56-60$ & $61-65$ & $66-70$ \\
\hline $\begin{array}{l}1.50 \\
1.55 \\
1.60 \\
1.65 \\
1.70 \\
1.75 \\
1.80 \\
1.85 \\
1.90\end{array}$ & $\begin{array}{c}3 \cdot 31 \\
2 \cdot 50 \\
3 \cdot 61 \\
2 \cdot 83 \\
3.91 \\
3 \cdot 16 \\
4 \cdot 21 \\
3 \cdot 50 \\
4 \cdot 51 \\
3 \cdot 80 \\
4 \cdot 81 \\
4 \cdot 10 \\
5 \cdot 12 \\
4 \cdot 41 \\
5 \cdot 42 \\
4 \cdot 69 \\
5 \cdot 72 \\
4.96\end{array}$ & $\begin{array}{c}3 \cdot 15 \\
2 \cdot 34 \\
3 \cdot 44 \\
2 \cdot 66 \\
3 \cdot 74 \\
2.98 \\
4 \cdot 04 \\
3 \cdot 31 \\
4 \cdot 33 \\
3 \cdot 61 \\
4 \cdot 63 \\
3 \cdot 90 \\
4 \cdot 93 \\
4 \cdot 20 \\
5 \cdot 23 \\
4 \cdot 46 \\
5 \cdot 52 \\
4 \cdot 73\end{array}$ & $\begin{array}{c}2 \cdot 99 \\
2 \cdot 17 \\
3 \cdot 28 \\
2 \cdot 49 \\
3 \cdot 57 \\
2 \cdot 80 \\
3 \cdot 86 \\
3 \cdot 12 \\
4 \cdot 15 \\
3 \cdot 42 \\
4 \cdot 44 \\
3 \cdot 70 \\
4 \cdot 74 \\
3 \cdot 99 \\
5 \cdot 03 \\
4 \cdot 22 \\
5 \cdot 32 \\
4 \cdot 50\end{array}$ & $\begin{array}{c}2 \cdot 83 \\
2 \cdot 00 \\
3 \cdot 11 \\
2 \cdot 32 \\
3 \cdot 40 \\
2 \cdot 63 \\
3 \cdot 68 \\
2.94 \\
3 \cdot 97 \\
3 \cdot 23 \\
4 \cdot 26 \\
3 \cdot 50 \\
4 \cdot 55 \\
3 \cdot 78 \\
4 \cdot 84 \\
3.99 \\
5 \cdot 12 \\
4 \cdot 27\end{array}$ & $\begin{array}{c}2 \cdot 67 \\
1.84 \\
2.94 \\
2.15 \\
3 \cdot 23 \\
2.46 \\
3 \cdot 50 \\
2.75 \\
3.79 \\
3.04 \\
4 \cdot 07 \\
3 \cdot 30 \\
4 \cdot 36 \\
3.57 \\
4.64 \\
3.75 \\
4.92 \\
4.05\end{array}$ & $\begin{array}{c}2 \cdot 51 \\
1 \cdot 67 \\
2 \cdot 78 \\
1 \cdot 99 \\
3 \cdot 06 \\
2 \cdot 28 \\
3 \cdot 33 \\
2 \cdot 57 \\
3 \cdot 61 \\
2 \cdot 85 \\
3 \cdot 89 \\
3 \cdot 10 \\
4 \cdot 17 \\
3 \cdot 36 \\
4 \cdot 45 \\
3 \cdot 52 \\
4 \cdot 72 \\
3.82\end{array}$ & $\begin{array}{c}2 \cdot 35 \\
1 \cdot 50 \\
2 \cdot 61 \\
1 \cdot 82 \\
2 \cdot 89 \\
2 \cdot 01 \\
3 \cdot 15 \\
2 \cdot 38 \\
3 \cdot 43 \\
2 \cdot 66 \\
3 \cdot 70 \\
2 \cdot 90 \\
3 \cdot 98 \\
3 \cdot 15 \\
4 \cdot 25 \\
3 \cdot 28 \\
4 \cdot 52 \\
3 \cdot 59\end{array}$ & $\begin{array}{c}2 \cdot 18 \\
1 \cdot 34 \\
2 \cdot 44 \\
1 \cdot 66 \\
2 \cdot 71 \\
1.93 \\
2.98 \\
2 \cdot 20 \\
3 \cdot 25 \\
2 \cdot 47 \\
3 \cdot 52 \\
2 \cdot 70 \\
3 \cdot 79 \\
2.94 \\
4 \cdot 05 \\
3.05 \\
4 \cdot 32 \\
3.37\end{array}$ & $\begin{array}{c}2 \cdot 02 \\
1 \cdot 18 \\
2 \cdot 28 \\
1 \cdot 50 \\
2 \cdot 54 \\
1 \cdot 76 \\
2 \cdot 81 \\
2 \cdot 02 \\
3 \cdot 07 \\
2 \cdot 28 \\
3 \cdot 34 \\
2 \cdot 50 \\
3 \cdot 60 \\
2 \cdot 73 \\
3 \cdot 86 \\
2 \cdot 82 \\
4 \cdot 12 \\
3 \cdot 15\end{array}$ & $\begin{array}{c}1.86 \\
1.01 \\
2.11 \\
1.33 \\
2.37 \\
1.58 \\
2.63 \\
1.84 \\
2.89 \\
2.09 \\
3.15 \\
2.30 \\
3.41 \\
2.52 \\
3.66 \\
2.58 \\
3.92 \\
2.92\end{array}$ \\
\hline
\end{tabular}

Upper row: mean values; lower row: lower values-95\% confidence limit. 


\section{DISCUSSION}

The Figure compares our results with those of the French and American studies. It can be seen firstly that as far as VC is concerned, the North American values are lower than both the Greek and, especially, the western European ones. In addition, their confidence interval being, in general, wider, the lower normal limits differ, in some cases, by almost one litre from the corres? ponding west European ones. This is not sur prising as it is well known that the latter suffe from an upward bias, but these values are used extensively in Europe and their comparisor? with other values may always be fruitful. Secondlye the Greek mean values of $\mathrm{FEV}_{1}$ are higher than those of the U.S. for younger ages ( 20 or 30 years) but the differences decrease progressively with agew (a)

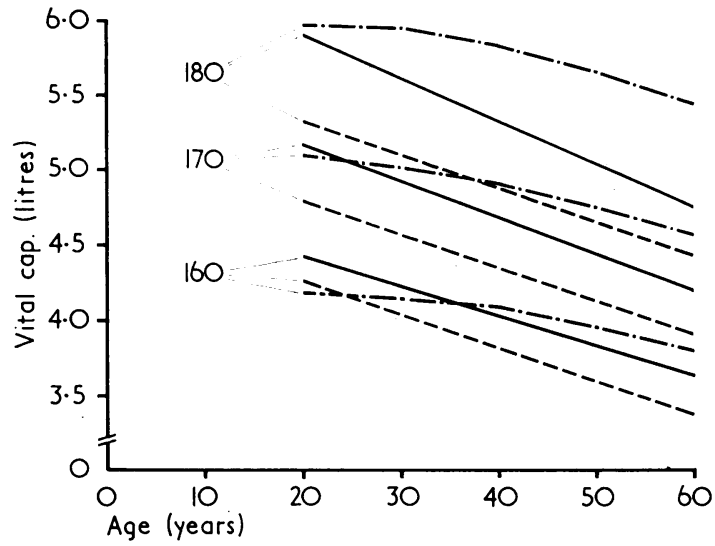

(b)

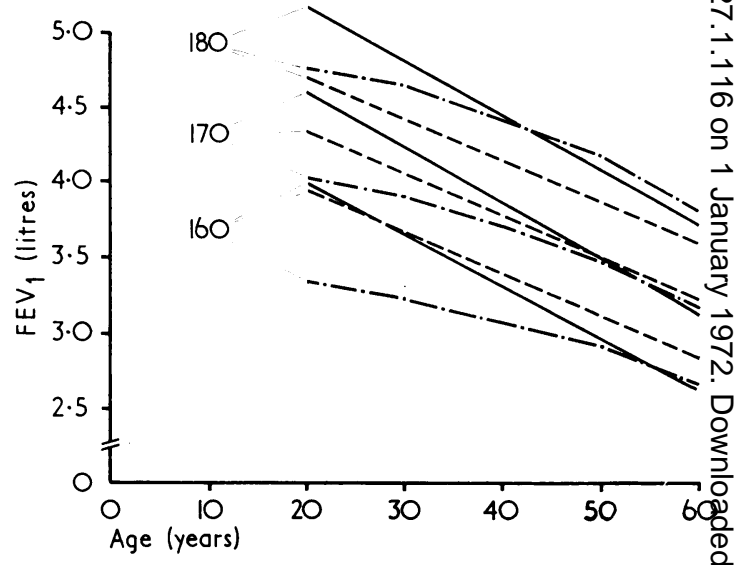

FIGURE. The relation of (a) vital capacity and (b) forced expiratory volume in 1 second $\left(F E V_{1}\right)$ to age for thre $\overrightarrow{\overrightarrow{8}}$ different heights $(160,170$, and $180 \mathrm{~cm})$. Data for subjects from the U.S.A. (---), France (----), and Greece (- - ).

VITAL CAPACITY: MEAN VALUES AND 95\% CONFIDENCE LIMIT

\begin{tabular}{|c|c|c|c|c|c|c|}
\hline \multirow{2}{*}{$\begin{array}{c}\text { Height } \\
\text { (m) }\end{array}$} & & \multirow[b]{2}{*}{20} & & \multicolumn{2}{|l|}{ Age Group } & \multirow[b]{2}{*}{$60-$} \\
\hline & & & $30-$ & $40-$ & $50-$ & \\
\hline $1 \cdot 60$ & $\begin{array}{l}\text { Greece } \\
\text { U.S.A. } \\
\text { Central Europe }\end{array}$ & $\begin{array}{l}4.43(3.63) \\
4.28(3.12) \\
4.20(3.49)\end{array}$ & $\begin{array}{l}4 \cdot 23(3.42) \\
4.06(2.90) \\
4 \cdot 18(3.47)\end{array}$ & $\begin{array}{l}4 \cdot 03(3.22) \\
3 \cdot 84(2 \cdot 68) \\
4 \cdot 10(3.40)\end{array}$ & $\begin{array}{l}3.83(3.01) \\
3.62(2.46) \\
3.97(3.30)\end{array}$ & $\begin{array}{l}3.63(2 \cdot 81) \\
3.40(2 \cdot 24) \\
3.81(3 \cdot 16)\end{array}$ \\
\hline $1 \cdot 70$ & $\begin{array}{l}\text { Greece } \\
\text { U.S.A. } \\
\text { Central Europe }\end{array}$ & $\begin{array}{l}5 \cdot 17(4 \cdot 41) \\
4 \cdot 80(3.64) \\
5.04(4 \cdot 18)\end{array}$ & $\begin{array}{l}4.93(4 \cdot 16) \\
4 \cdot 58(3 \cdot 42) \\
5 \cdot 01(4 \cdot 16)\end{array}$ & $\begin{array}{l}4 \cdot 68(3 \cdot 89) \\
4 \cdot 36(3 \cdot 20) \\
4 \cdot 91(4 \cdot 08)\end{array}$ & $\begin{array}{l}4.43(3.62) \\
4.14(2.98) \\
4.77(3.96)\end{array}$ & $\begin{array}{l}4 \cdot 19(3 \cdot 36) \\
3 \cdot 92(2 \cdot 76) \\
4 \cdot 57(3 \cdot 79)\end{array}$ \\
\hline $1 \cdot 80$ & $\begin{array}{l}\text { Greece } \\
\text { U.S.A. } \\
\text { Central Europe }\end{array}$ & $\begin{array}{l}5.91(5 \cdot 15) \\
5 \cdot 32(4 \cdot 16) \\
5.98(4 \cdot 96)\end{array}$ & $\begin{array}{l}5.62(4.84) \\
5.10(3.94) \\
5.95(4.93)\end{array}$ & $\begin{array}{l}5 \cdot 32(4 \cdot 50) \\
4 \cdot 88(3 \cdot 72) \\
5 \cdot 83(4 \cdot 84)\end{array}$ & $\begin{array}{l}5 \cdot 03(4 \cdot 16) \\
4 \cdot 66(3 \cdot 50) \\
5 \cdot 66(4 \cdot 70)\end{array}$ & $\begin{array}{l}4 \cdot 74(3 \cdot 83) \\
4 \cdot 44(3 \cdot 28) \\
5 \cdot 42(4 \cdot 50)\end{array}$ \\
\hline
\end{tabular}

FEV $_{1}:$ MEAN VALUES AND $95 \%$ CONFIDENCE LIMIT

\begin{tabular}{|c|c|c|c|c|c|c|}
\hline \multirow{2}{*}{$\begin{array}{l}\text { Height } \\
\text { (m) }\end{array}$} & & \multicolumn{5}{|c|}{ Age Group } \\
\hline & & 20 & 30 & 40 & 50 & 60 \\
\hline $1 \cdot 60$ & $\begin{array}{l}\text { Greece } \\
\text { U.S.A. } \\
\text { Central Europe }\end{array}$ & $\begin{array}{l}3.99(3.24) \\
3.97(2.93) \\
3.35(2.71)\end{array}$ & $\begin{array}{l}3.65(2.89) \\
3.69(2.65) \\
3.26(2.64)\end{array}$ & $\begin{array}{l}3.31(2 \cdot 54) \\
3.41(2 \cdot 37) \\
3 \cdot 10(2 \cdot 52)\end{array}$ & $\begin{array}{l}2.99(2.20) \\
3.13(2.09) \\
2.92(2.37)\end{array}$ & $\begin{array}{l}2.64(1.84) \\
2.85(1.81) \\
2.67(2.16)\end{array}$ \\
\hline $1 \cdot 70$ & $\begin{array}{l}\text { Greece } \\
\text { U.S.A. } \\
\text { Central Europe }\end{array}$ & $\begin{array}{l}4.60(3.89) \\
4.34(3 \cdot 30) \\
4.02(3 \cdot 26)\end{array}$ & $\begin{array}{l}4.24(3.52) \\
4.06(3.02) \\
3.91(3.17)\end{array}$ & $\begin{array}{l}3.88(3.14) \\
3.78(2.74) \\
3.72(3.01)\end{array}$ & $\begin{array}{l}3.52(2.76) \\
3.50(2.46) \\
3.50(2.83)\end{array}$ & $\begin{array}{l}3 \cdot 16(2 \cdot 38) \\
3 \cdot 22(2 \cdot 18) \\
3 \cdot 20(2 \cdot 59)\end{array}$ \\
\hline $1 \cdot 80$ & $\begin{array}{l}\text { Greece } \\
\text { U.S.A. } \\
\text { Central Europe }\end{array}$ & $\begin{array}{l}5.21(4.50) \\
4.71(3.67) \\
4.77(3.86)\end{array}$ & $\begin{array}{l}4.83(4 \cdot 10) \\
4.43(3.39) \\
4.64(3.76)\end{array}$ & $\begin{array}{l}4.46(3.69) \\
4.15(3.11) \\
4.42(3.58)\end{array}$ & $\begin{array}{l}4.07(3.26) \\
3.87(2 \cdot 83) \\
4.16(3.37)\end{array}$ & $\begin{array}{l}3.70(2.85) \\
3.59(2.55) \\
3.80(3.08)\end{array}$ \\
\hline
\end{tabular}


Thus at 50 or 60 years the Greek and U.S. values are almost the same. It is nevertheless worthwhile noticing that, in general, the $F E V_{1}$ values for the three populations do not differ significantly.

Our findings suggest, therefore, that the Greek mean values are slightly higher than those for the U.S. The reason is not clear. Possibly our criteria of selection were more severe, as we selected subjects who had had no chest illness within the previous three years, whereas Kory et al. (1961) discarded only those subjects with a history of chest disease during the six months preceding the test. Another possible reason may be that our subjects were living in areas where air pollution is considered to be low. Recent studies (Alivizatos, Bazas, Alexopoulos, and Verikokakis, 1965, 1968) have shown that heavy air pollution is limited to the central commercial area of Athens where none of our subjects was living permanently. On the other hand, tobacco consumption cannot be taken into consideration as neither this work nor that of Kory et al. distinguished between smokers and non-smokers. Moreover, its influence on spirometric values is uncertain (Cotes et al., 1964).

Although there is serious statistical criticism of the use of lower limits of normal as reference points for lung function assessment ${ }^{1}$, the figures given in Table II seem to be valuable in clinical practice. Moreover, the lower limits have been proved to be useful in daily clinical practice and this is the main reason for their continued use. 1International Symposium on Respiratory Physiology, Sassari, May
1969
Many thanks are due to Dr. J. E. Cotes for considerable help throughout this study.

\section{REFERENCES}

Alivizatos, G. P., Bazas, B. N., Alexopoulos, G., and Verikokakis, E. (1965). The problem of air pollution in the city and greater Athens. Arch. Hyg. (Athinae), 15, 329. (In Greek; Eng. summary, p. 392).

(1968). The problem of air pollution in Athens and surrounding territory. Part II. Arch. Hyg., 18, 1. (In Greek; Eng. summary, p. 20).

Baldwin, E. de F., Cournand, A., and Richards, D. W. (1948). Pulmonary insufficiency. I. Physiological classification, clinical methods of analysis, standard values in normal subjects. Medicine (Baltimore), 27, 243.

Berglund, E., Birath, G., Bjure, J., Grimby, G., Kjellmer, I., Sandqvist, L., and Söderholm, B. (1963). Spirometric studies in normal subjects. I. Forced expirograms in subjects between 7 and 70 years of age. Acta med. scand., 173, 185.

Commission des Communautés Européennes. Tables de références pour les examens spirographiques. Luxembourg, 1967.

Cotes, J. E. (1965). Lung Function. Blackwell, Oxford.

- Higgins, I. T. T., Rossiter, C. R., and Wilton, C. (1964) (unpublished). Normal values for the forced expiratory volume in British males analysed by age, height and tobacco consumption. Account of surveys in different parts of the U.K. by the Pneumoconiosis Research Unit of M.R.C.

Kory, R. C., Callahan, R., Boren, H. G., and Syner, J. C. (1961). The Veterans Administration-Army cooperative study of pulmonary function. I. Clinical spirometry in normal men. Amer. J. Med., 30, 243.

McKerrow, C. B., McDermott, M., and Gilson, J. C. (1960). A spirometer for measuring the forced expiratory volume with a simple calibrating device. Lancet, 1, 149. 